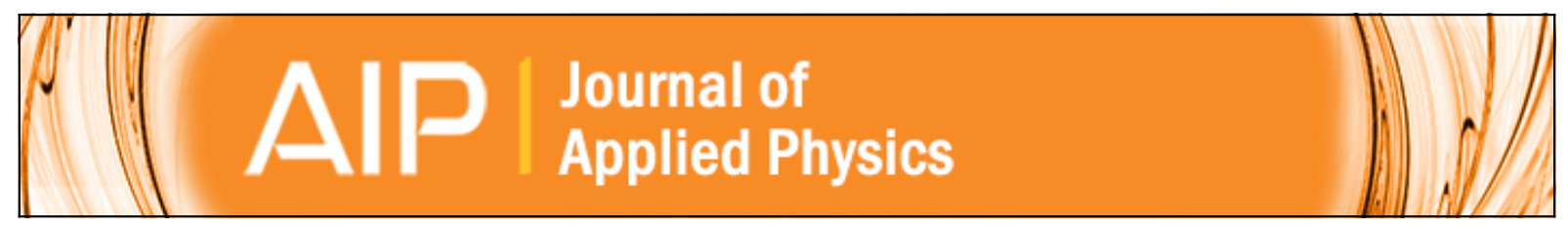

\title{
Effect of irradiation temperature and ion flux on electrical isolation of GaN
}

S. O. Kucheyev, H. Boudinov, J. S. Williams, C. Jagadish, and G. Li

Citation: Journal of Applied Physics 91, 4117 (2002); doi: 10.1063/1.1455154

View online: http://dx.doi.org/10.1063/1.1455154

View Table of Contents: http://scitation.aip.org/content/aip/journal/jap/91/7?ver=pdfcov

Published by the AIP Publishing

Articles you may be interested in

Interaction of defects and $\mathrm{H}$ in proton-irradiated $\mathrm{GaN}(\mathrm{Mg}, \mathrm{H})$

J. Appl. Phys. 97, 093517 (2005); 10.1063/1.1883309

Ion-irradiation-induced porosity in GaSb

Appl. Phys. Lett. 86, 131920 (2005); 10.1063/1.1896428

Electrical characteristics of $\mathrm{GaN}$ implanted with $\mathrm{Si}+$ at elevated temperatures

Appl. Phys. Lett. 86, 112108 (2005); 10.1063/1.1884744

Electrical isolation of $\mathrm{n}$ - and $\mathrm{p}-\operatorname{In} 0.53 \mathrm{Ga} 0.47$ As epilayers using ion irradiation

J. Appl. Phys. 94, 6616 (2003); 10.1063/1.1619567

Electrical isolation of n-type GaAs layers by proton bombardment: Effects of the irradiation temperature J. Appl. Phys. 84, 4757 (1998); 10.1063/1.368817

\section{AIP $\left.\right|_{\text {APL Photonics }}$}

APL Photonics is pleased to announce Benjamin Eggleton as its Editor-in-Chief

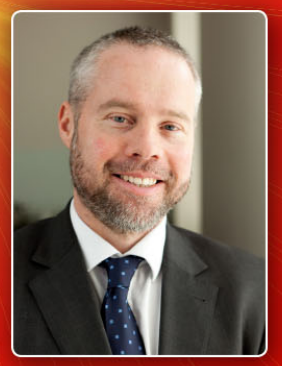




\title{
Effect of irradiation temperature and ion flux on electrical isolation of GaN
}

\author{
S. O. Kucheyeva) \\ Department of Electronic Materials Engineering, Research School of Physical Sciences and Engineering, \\ The Australian National University, Canberra, ACT 0200, Australia \\ H. Boudinov \\ Instituto de Física, UFRGS, Porto Alegre, R.S., Brazil
}

J. S. Williams and C. Jagadish

Department of Electronic Materials Engineering, Research School of Physical Sciences and Engineering, The Australian National University, Canberra, ACT 0200, Australia

G. Li

Ledex Corporation, No. 9, Ta-Yio First St., Ta-Fa Industrial District, Kaohsiung County, Taiwan, Republic of China

(Received 28 November 2001; accepted for publication 6 January 2002)

\begin{abstract}
We study the evolution of sheet resistance of $n$-type GaN epilayers irradiated with $\mathrm{MeV}^{1} \mathrm{H}$ and ${ }^{12} \mathrm{C}$ ions. Results show that both implantation temperature (varied from 77 up to $423 \mathrm{~K}$ ) and ion beam flux affect the process of electrical isolation in the case of irradiation with ${ }^{12} \mathrm{C}$ ions. This behavior is consistent with significant dynamic annealing occurring in $\mathrm{GaN}$ during $\mathrm{MeV}$ light-ion bombardment, which suggests a scenario where the centers responsible for electrical isolation are defect clusters or anti-site-related defects. Dynamic annealing causes simple ion-beam-generated Frenkel pairs to annihilate (or cluster) during irradiation at liquid nitrogen temperature and above. These beam-flux and irradiation-temperature effects are not observed during bombardment with lighter ${ }^{1} \mathrm{H}$ ions, which produce very dilute collision cascades. A qualitative model is proposed to explain temperature and flux effects in $\mathrm{GaN}$ in the $\mathrm{MeV}$ light-ion bombardment regime used for electrical isolation. (C) 2002 American Institute of Physics. [DOI: 10.1063/1.1455154]
\end{abstract}

\section{INTRODUCTION}

Gallium nitride is a very promising material for hightemperature/high-power electronic devices. ${ }^{1}$ In the fabrication of such GaN-based devices, ion irradiation is an ideal processing tool for electrical isolation. Indeed, as has previously been shown, ${ }^{2-7}$ ion bombardment can render GaN highly resistive, which can be used for electrical isolation of closely spaced devices. Moreover, electrical isolation in $\mathrm{GaN}$ can be stable to subsequent rapid thermal annealing at temperatures as high as $\sim 1173 \mathrm{~K},{ }^{7}$ which is important for potential applications of this material for high-temperature electronics.

Although the possibility of electrical isolation in $\mathrm{GaN}$ by ion bombardment has previously been demonstrated, ${ }^{2-7}$ systematic studies are still lacking in the literature. In particular, the influence of irradiation parameters (such as ion mass, energy, sample temperature, and beam flux) has not been established. Such systematic studies are needed not only for a choice of irradiation conditions necessary for an effective electrical isolation of GaN-based devices but also for a better understanding of ion-beam processes responsible for isolation.

Previous investigation of the effect of implant conditions on the structural characteristics of $\mathrm{GaN}$ bombarded with $\mathrm{keV}$ ions has revealed very efficient dynamic annealing (i.e., defect migration and interaction) processes in this material at

${ }^{a)}$ Electronic mail: sergei.kucheyev@anu.edu.au liquid nitrogen temperature and above. ${ }^{8}$ Although most of ion-beam-generated Frenkel pairs experience annihilation, dynamic annealing is never perfect, and stable lattice disorder accumulates with increasing ion dose. As a result, dynamic annealing causes stable damage to depend on collision cascade density, irradiation temperature, and beam flux. Furthermore, $\mathrm{keV}$ ion bombardment leads to the formation of a range of complex stable defects, many of which can be imaged by transmission electron microscopy as extended defects. For example, even irradiation with relatively light ${ }^{12} \mathrm{C}$ ions at $\mathrm{keV}$ energies leads to the formation of stable extended defects and exhibits a dependence of the damage buildup on irradiation temperature and beam flux. ${ }^{8}$

Although dynamic annealing has been found to have a dramatic influence on residual damage in GaN bombarded with $\mathrm{keV}$ ions, particularly heavy ions, its effect on electrical isolation using low-dose $\mathrm{MeV}$ light ions is not obvious. ${ }^{9}$ Indeed, the effective diffusivity of irradiation-produced defects (which controls the level of dynamic annealing) may depend on ion dose and collision cascade density due to, for example, (i) processes of defect trapping and de-trapping during migration (which depend on the defect concentration in the lattice), (ii) possible dependence of defect migration parameters on the position of the chemical potential, and (iii) radiation-enhanced migration. It is, therefore, imperative that dynamic annealing (and associated irradiation-temperature and beam-flux dependencies) be investigated in the case of low-dose $\mathrm{MeV}$ light-ion bombardment used for electrical isolation. 
In this article, we study the effects of irradiation temperature, ion beam flux, and collision cascade density on electrical isolation of $\mathrm{GaN}$. Results reveal that irradiation temperature and ion beam flux indeed affect electrical isolation in GaN. This suggests that the centers responsible for isolation are defect clusters or anti-site-related defects, whereas ion-beam-generated Frenkel pairs (i.e., simple vacancies and interstitials in both sublattices in all possible charge states) in $\mathrm{GaN}$ are mobile during ion irradiation at liquid nitrogen temperature and above.

\section{EXPERIMENT}

The $n$-type wurtzite GaN film used in this study was $\sim 2$ $\mu \mathrm{m}$ thick (an $\sim 1.5 \mu \mathrm{m}$ conductive layer over an $\sim 0.5 \mu \mathrm{m}$ undoped GaN buffer), epitaxially grown on a $c$-plane sapphire substrate by metalorganic chemical vapor deposition (MOCVD) in a rotating disk reactor at Ledex Corporation. As-grown samples cut from this wafer had a roomtemperature free electron concentration of $\sim 3 \times 10^{17} \mathrm{~cm}^{-3}$, an effective Hall mobility of $\sim 500-600 \mathrm{~cm}^{2} \mathrm{~V}^{-1} \mathrm{~s}^{-1}$, and a sheet resistance of $\sim 300 \Omega /$ sq. Resistors of a rectangular geometry were prepared. Ohmic contacts were formed by electron-beam evaporation of Ti/Al/Au $(40 \mathrm{~nm} / 120 \mathrm{~nm} / 500$ $\mathrm{nm}$ ) and subsequent annealing at $1173 \mathrm{~K}$ for $30 \mathrm{~s}$ in an argon ambient at atmospheric pressure. Because the thickness of the contact layer was not sufficient to prevent the isolation of the underlying $\mathrm{GaN}$ regions by $\mathrm{MeV}$ light-ion irradiation, additional masking with an aluminum foil of the contact regions was necessary.

These $\mathrm{GaN}$ resistors were irradiated at temperatures from 77 up to $423 \mathrm{~K}$ with $0.6 \mathrm{MeV}{ }^{1} \mathrm{H}^{+}$or $6.6 \mathrm{MeV}^{12} \mathrm{C}^{3+}$ ions to doses in the range from $1 \times 10^{10}$ to $2 \times 10^{15} \mathrm{~cm}^{-2}$ using an ANU 1.7 MV tandem accelerator (NEC, 5SDH-4) or the UFRGS 3 MV HVEE tandem accelerator. Such ion energies were chosen to place the damage peak in the sapphire substrate, beyond the GaN layer. During bombardment, samples were tilted by $7^{\circ}$ off the surface normal direction to minimize channeling. An average scanned ion beam flux was kept constant during implantation to different doses with $\mathrm{H}$ or $\mathrm{C}$ ions to study the flux effect. It is important to note that, during such measurements, ion beam flux was varied by changing beam current from the ion source, without changing beam focusing (i.e., beam spot size) and beam scanning parameters. Sheet resistance $\left(R_{s}\right)$ was measured in situ after each dose step using a Keithley 619 electrometer. In cases where irradiation was performed at low or elevated temperatures, samples were brought to room temperature before resistance measurements. Selected samples were also studied by Hall effect measurements.

\section{RESULTS AND DISCUSSION}

Figure 1 shows the evolution of $R_{s}$ of $\mathrm{GaN}$ resistors irradiated with $6.6 \mathrm{MeV} \mathrm{C}$ ions at three different temperatures. An increase in $R_{s}$ with increasing ion dose observed in Fig. 1 is due to the degradation of carrier mobility and the trapping of charge carriers at deep centers associated with irradiation-produced lattice damage. ${ }^{10}$ It is clearly seen from Fig. 1 that irradiation temperature affects the process of elec-

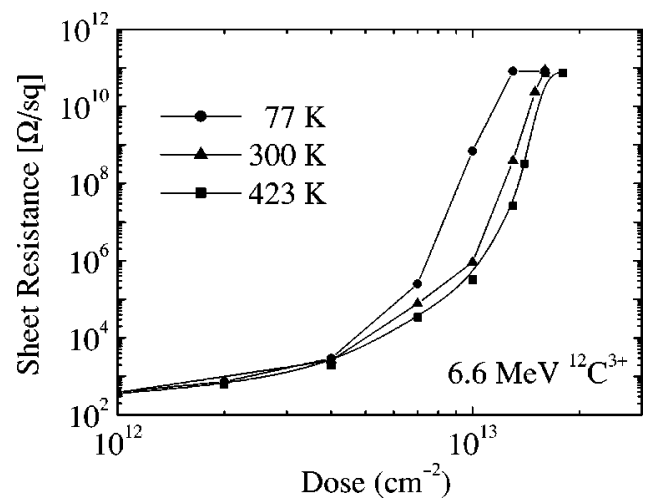

FIG. 1. Dose dependence of sheet resistance of GaN samples irradiated with 6.6 MeV C ions with a beam flux of $1 \times 10^{11} \mathrm{~cm}^{-2} \mathrm{~s}^{-1}$ at different temperatures, as indicated in the legend.

trical isolation in GaN. Indeed, Fig. 1 reveals that the ion dose required to achieve a given value of $R_{s}$ increases with increasing implantation temperature. This behavior is consistent with dynamic annealing during irradiation, whereby an increase in irradiation temperature enhances the migration, annihilation, and agglomeration processes of ion-beamgenerated point defects. With increasing irradiation temperature, more migrating defects experience annihilation, and fewer point defects form the stable and electrically active complexes responsible for electrical isolation.

Interestingly, our results show that the density of collision cascades influences the magnitude of the irradiationtemperature effect. For example, in contrast to the case of ${ }^{12} \mathrm{C}$ ions shown in Fig. 1, results on electrical isolation of GaN using very light $0.6 \mathrm{MeV}{ }^{1} \mathrm{H}$ ions with a beam flux of $3 \times 10^{10} \mathrm{~cm}^{-2} \mathrm{~s}^{-1}$ reveal a negligible effect of sample temperature for temperatures varied from 77 up to $423 \mathrm{~K}$ (the figure is not shown). We discuss the significance of this result below. However, here it should be noted that the maximum irradiation temperature which can be used in dynamic annealing studies is limited by the effects of postirradiation annealing. Although isolation produced in $\mathrm{GaN}$ by large ion doses (when $R_{s}$ reaches the maximum) is stable to rapid thermal annealing at temperatures as high as $\sim 1173 \mathrm{~K}$, ${ }^{7}$ isolation produced by lower doses (where $R_{s}$ is several orders of magnitude lower than the maximum isolation value) is not thermally stable at such high temperatures. Indeed, Fig. 2 shows results of isochronal annealing of a $\mathrm{GaN}$ sample bombarded with very light $0.6 \mathrm{MeV}{ }^{1} \mathrm{H}$ ions to a dose of 2.4 $\times 10^{14} \mathrm{~cm}^{-2}$. It is seen from Fig. 2 that, even for rather low doses of very light ${ }^{1} \mathrm{H}$ ions (when $R_{s}$ is $\sim$ six orders of magnitude lower than its maximum value), electrical isolation is stable to postirradiation thermal annealing at temperatures below $\sim 500 \mathrm{~K}$. However, for annealing temperatures above $\sim 500 \mathrm{~K}$, Fig. 2 reveals a pronounced increase in sheet electron density and effective Hall mobility (and an associated decrease in $R_{s}$ ) due to annealing of ion-beam-produced defects. Hence, in the present study, the maximum irradiation temperature has been chosen below $500 \mathrm{~K}$, to ensure that little thermal annealing of the centers responsible for electrical isolation occurs during irradiation. 


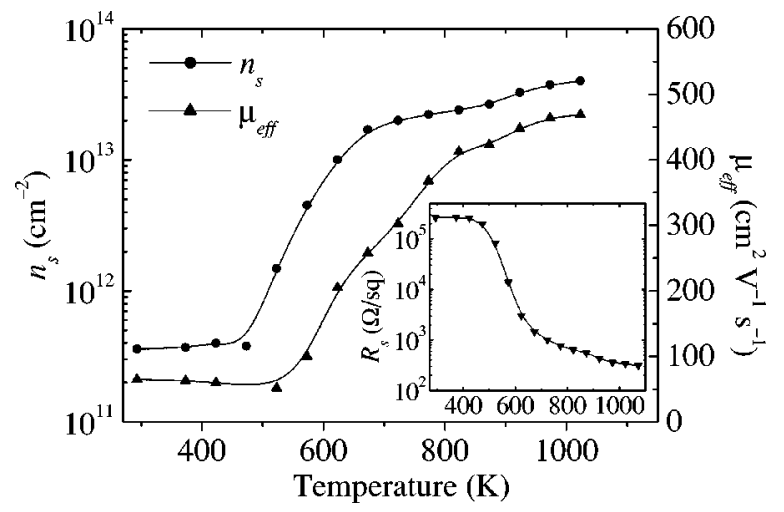

FIG. 2. Dependencies of sheet electron density $n_{s}$ (the left scale), effective Hall mobility $\mu_{\text {eff }}$ (the right scale), and sheet resistance $R_{s}$ (the inset) on isochronal annealing temperature for a $\mathrm{GaN}$ sample implanted at room temperature with $0.6 \mathrm{MeV} \mathrm{H}$ ions with a beam flux of $4 \times 10^{11} \mathrm{~cm}^{-2} \mathrm{~s}^{-1}$ to a dose of $2.4 \times 10^{14} \mathrm{~cm}^{-2}$. Each postirradiation annealing step was performed for $1 \mathrm{~min}$ in an argon ambient at atmospheric pressure. Results of Hall effect measurements.

Shown in Fig. 3 is the evolution of $R_{s}$ of GaN resistors bombarded with $6.6 \mathrm{MeV} \mathrm{C}$ ions at room temperature with different ion beam fluxes. It is seen from Fig. 3 that the isolation process depends on ion beam flux. ${ }^{11}$ Indeed, the ion dose required to achieve a given value of $R_{s}$ decreases with increasing beam flux. This so-called "normal" flux effect (when, with increasing beam flux, the level of ion-beamproduced damage also increases) is again consistent with the fact that dynamic annealing processes affect electrical isolation in $\mathrm{GaN}$. Indeed, an increase in beam flux decreases the average time interval between collision cascades which spatially overlap. Increasing beam flux increases the generation rate of point defects and, hence, enhances the rate of interactions between mobile defects (generated in different collision cascades). Thus, as discussed later, increased beam flux aids the formation of stable defect clusters that are responsible for electrical isolation.

The influence of collision cascade density on the flux effect is illustrated in Fig. 4, which shows the evolution of $R_{S}$ of $\mathrm{GaN}$ resistors irradiated at room temperature with different beam fluxes of very light $0.6 \mathrm{MeV}{ }^{1} \mathrm{H}$ ions (which produce very dilute collision cascades). Figure 4 reveals a neg-

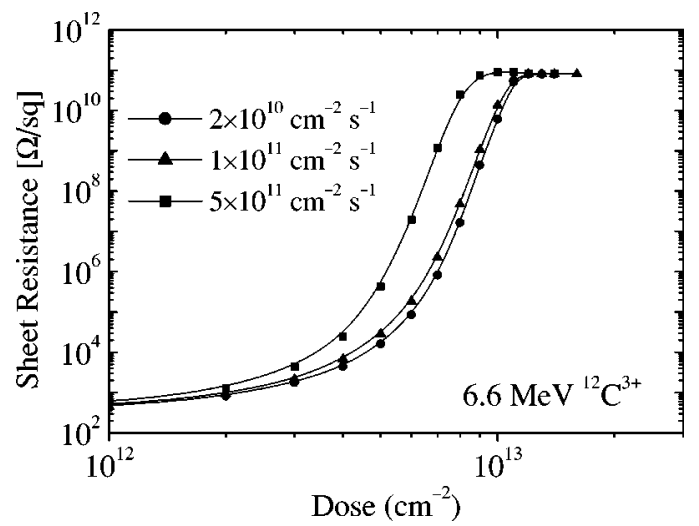

FIG. 3. Dose dependence of sheet resistance of GaN samples irradiated with 6.6 $\mathrm{MeV} \mathrm{C}$ ions at room temperature with different values of beam flux, as indicated in the legend.

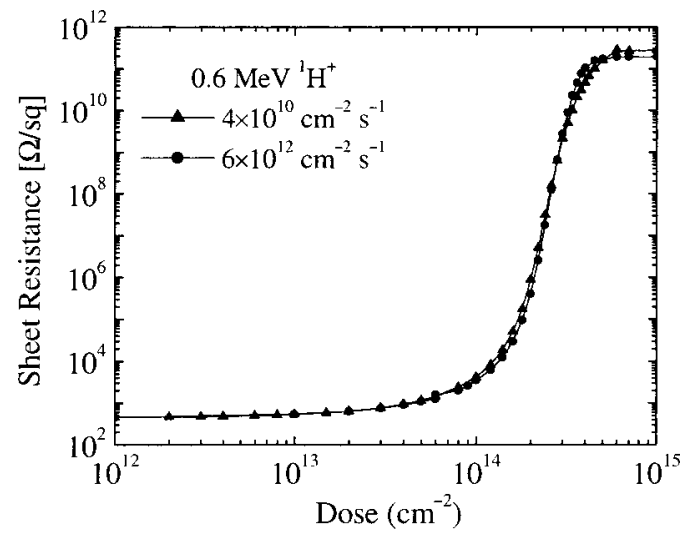

FIG. 4. Dose dependence of sheet resistance of GaN samples irradiated with $0.6 \mathrm{MeV} \mathrm{H}$ ions at room temperature with different values of beam flux, as indicated in the legend.

ligible beam flux effect in this case. Hence, both beam-flux and irradiation-temperature dependencies of complex defect interaction processes appear to be controlled by the density of collision cascades. We discuss these processes and differences between $\mathrm{H}$ and $\mathrm{C}$ cases in more detail below.

It is clear that the effectiveness of dynamic annealing depends on the defect generation rate [atomic displacements $/\left(\mathrm{cm}^{3} \mathrm{~s}\right)$ ], which is determined by ion mass, energy, and beam flux. In the first approximation, the flux effect can be related to an effective time constant which indicates the time it takes for defects created in a collision cascade to stabilize. Indeed, although the ballistic formation of a collision cascade by an impinging ion occurs in a very short time interval $(\sim 1 \mathrm{ps})$, cascade stabilization involving processes of migration and interaction of point defects proceeds for much longer times. This time constant, determined by the effective diffusivity of defects and defect interaction processes, obviously depends on both material properties and implant conditions (such as implantation temperature, beam flux, and collision cascade sizes and densities). In some cases, this time constant can be of the order of a second. ${ }^{12}$ For a pronounced flux effect, it is important that such a time constant be comparable with the average time interval between collision cascades that spatially overlap. It is expected that the flux effect vanishes in cases of either very low or very high rates of defect generation, which corresponds to cases of either (i) low cascade densities and/or low values of beam flux or (ii) high cascade densities and/or high values of beam flux, respectively.

The above scenario is consistent with our experimental data showing a negligible flux effect in the case of very light $0.6 \mathrm{MeV}{ }^{1} \mathrm{H}$ ions (Fig. 4) and a measurable flux effect for irradiation with heavier (but still relatively light) $6.6 \mathrm{MeV}$ ${ }^{12} \mathrm{C}$ ions (Fig. 3). The above results also show that the dependence of electrical isolation on irradiation temperature, controlled by the density of collision cascades, vanishes in the case of very light $0.6 \mathrm{MeV}{ }^{1} \mathrm{H}$ ions. This is attributed to the fact that, in the case of very dilute collision cascades produced by very light ions, point defects are very mobile even at liquid nitrogen temperature, resulting in a fast stabilization of collision cascades. In this case, an increase in 
irradiation temperature to $423 \mathrm{~K}$ is not expected to significantly change the number of stable defect complexes formed during ion bombardment, which is consistent with our experimental data. In contrast to this case of very light ions, for heavier ${ }^{12} \mathrm{C}$ ions, when collision cascades are much denser and the interaction of point defects created in different overlapping collision cascades occurs (as indicated by the presence of the flux effect), irradiation temperature affects the rate and nature of defect interaction processes. Hence, in such a case, implantation temperature influences the nature and magnitude of the stable defect complexes responsible for electrical isolation (see Fig. 1). These results indicate that, for a material subject to substantial dynamic annealing such as $\mathrm{GaN}$, an effective electrical isolation requires a careful consideration of all implant conditions (i.e., ion mass, energy, beam flux, and irradiation temperature).

Finally, a comment should be made on the nature of defects responsible for electrical isolation in GaN. The irradiation-temperature and beam-flux dependencies revealed in this study suggest that the centers responsible for isolation are not simple Frenkel pairs but the product of defect migration and interaction processes. The more complex nature of defects responsible for isolation in $\mathrm{GaN}$ is also strongly supported by the fact that these defect complexes have quite large dissociation energies, as revealed by their large thermal stability. Indeed, simple Frenkel pairs are expected to anneal out using much smaller thermal budgets than those necessary to recover $R_{s}$ in $\mathrm{GaN}(>1173 \mathrm{~K})$. Possible candidates for defect complexes responsible for isolation in $\mathrm{GaN}$ are (i) interstitial or vacancy based defect clusters, (ii) complexes of defects with lattice impurities, and (iii) anti-site-related defects. Our present experimental data cannot give preference to any of these defect types. Moreover, the processes of migration, clustering, and dissociation of point defect complexes in $\mathrm{GaN}$ have not yet been studied theoretically. Hence, at present, additional both experimental and theoretical studies are needed to identify defect types controlling the technologically important process of electrical isolation in GaN.

\section{CONCLUSIONS}

In conclusion, we have studied the effect of irradiation temperature, beam flux, and collision cascade density on electrical isolation of $n$-type $\mathrm{GaN}$ by $\mathrm{MeV}$ light-ion irradiation. Results have revealed the important role of dynamic annealing processes in $\mathrm{GaN}$ during $\mathrm{MeV}$ light-ion bombardment used for electrical isolation. This suggests that the centers responsible for electrical isolation are defect complexes or anti-site-related defects, whereas ion-beam-generated simple Frenkel pairs in GaN are mobile and unstable during irradiation at liquid nitrogen temperature and above. The influence of irradiation temperature and beam flux revealed in the present study should be taken into account for choosing implant conditions necessary for an effective electrical isolation of GaN-based devices.

\footnotetext{
${ }^{1}$ See, for example, a review by S. J. Pearton, J. C. Zolper, R. J. Shul, and F. Ren, J. Appl. Phys. 86, 1 (1999).

${ }^{2}$ S. C. Binari, H. B. Dietrich, G. Kelner, L. B. Rowland, K. Doverspike, and D. K. Wickenden, J. Appl. Phys. 78, 3008 (1995).

${ }^{3}$ S. J. Pearton, C. B. Vartuli, J. C. Zolper, C. Yuan, and R. A. Stall, Appl. Phys. Lett. 67, 1435 (1995).

${ }^{4}$ S. J. Pearton, R. G. Wilson, J. M. Zavada, J. Han, and R. J. Shul, Appl. Phys. Lett. 73, 1877 (1998).

${ }^{5}$ C. Uzan-Saguy, J. Salzman, R. Kalish, V. Richter, U. Tish, S. Zamir, and S. Prawer, Appl. Phys. Lett. 74, 2441 (1999).

${ }^{6}$ X. A. Cao, S. J. Pearton, G. T. Dang, A. P. Zhang, F. Ren, R. G. Wilson, and J. M. Van Hove, J. Appl. Phys. 87, 1091 (2000).

${ }^{7}$ H. Boudinov, S. O. Kucheyev, J. S. Williams, C. Jagadish, and G. Li, Appl. Phys. Lett. 78, 943 (2001).

${ }^{8}$ See, for example, a recent review by S. O. Kucheyev, J. S. Williams, and S. J. Pearton, Mater. Sci. Eng., R. 33, 51 (2001).

${ }^{9}$ It should be noted that typical ion irradiation studies of structural characteristics and electrical isolation are performed in very different ion dose regimes. Indeed, electrical isolation involves ion doses which result in the number of atomic displacements comparable with the concentration of free carriers (usually $\sim 10^{15}-10^{18} \mathrm{~cm}^{-3}$ ), whereas typical studies of structural characteristics use ion doses resulting in the number of atomic displacements comparable with the atomic concentration of the material $\left(\sim 10^{23} \mathrm{~cm}^{-3}\right)$

${ }^{10}$ See, for example, a review by S. J. Pearton, Mater. Sci. Rep. 4, 313 (1990), and references therein.

${ }^{11}$ Note that, in Figs. 1 and 3, isolation curves shown by triangles do not overlap despite the apparently identical implant conditions used. This is due to different beam focusing (i.e., ion beam spot size) used to irradiate samples shown in Figs. 1 and 3. This result clearly illustrates that not only averaged beam flux but also instantaneous beam flux (determined by ion current in the spot mode and ion beam spot size) should be taken into account during flux effect measurements.

${ }^{12}$ J. Linnros and G. Holmen, J. Appl. Phys. 62, 4737 (1987).
} 